\title{
INCREASED EXPRESSION OF PENTRAXIN 3 IN PLACENTAL TISSUES FROM PATIENTS WITH UNEXPLAINED RECURRENT PREGNANCY LOSS
}

\author{
Zeybek $\mathrm{S}^{1, *}$, Tepeli $\mathrm{E}^{2}$, Cetin $\mathrm{GO}^{3}$, Caner $\mathrm{V}^{3}$, Senol $\mathrm{H}^{4}$, Yildirim $\mathrm{B}^{2}$, Bagci $\mathrm{G}^{3}$ \\ *Corresponding Author: Selcan Zeybek, M.D., Department of Medical Genetics, Erzurum Regional \\ Training and Research Hospital, Cat Volu Street, 25070, Erzurum, Turkey. Tel.: +90-506-399-2644. \\ Fax: +90-442-232-5025. E-mail: selcankesan@yahoo.com
}

\begin{abstract}
Pentraxin 3 (PTX3), a prototypical member of the long pentraxin subfamily, is a evolutionarily conserved multimeric pattern recognition receptor involved in the humoral component of the innate immune system. Pentraxin 3 is released when tissue is stressed or damaged, and interacts with many different ligands. Pentraxin 3 exerts a pivotal role both as a regulator and as an indicator of inflammatory response in the pathogenesis of many diseases such as sepsis, vasculitis and preeclampsia. Uncontrolled inflammatory response is considered a major cause of unexplained recurrent pregnancy loss (URPL). We determined the $P T X 3$ messenger ribonucleic acid (mRNA) and protein expression levels in placental tissues from 50 women with URPL, and made comparison with those in 50 age-matched control subjects. In quantitative real-time polymerase chain reaction (qRT-PCR) and immunohistochemistry analyses, PTX3 mRNA and protein levels, respectively, were significantly increased in URPL patients compared with their respective controls $(p=0.0001)$. Although no significant correlations were identified between PTX3 expression levels and clinical parameters such as maternal age, numbers of previous pregnancy losses, and gestational age at miscarriage, PTX3 mRNA expression was significantly higher in patients with no live births than in women with previous live births $(p=0.0001)$. Our study suggests that tissue-specific expression of PTX 3 is

\footnotetext{
$\overline{{ }^{1} \text { Department of Medical Genetics, Erzurum Regional Training and }}$ Research Hospital, Erzurum, Turkey

${ }^{2}$ Private Clinic, Denizli, Turkey

${ }^{3}$ Department of Medical Genetics, Faculty of Medicine, Pamukkale University, Denizli, Turkey

${ }^{4}$ Department of Biostatistics, Faculty of Medicine, Pamukkale University, Denizli, Turkey
}

associated with URPL. Further larger studies are required to determine whether PTX3 expression can be used as a biomarker to manage URPL in routine clinical practice.

Keywords: Inflammation; Pentraxin 3 (PTX3); Placental expression; Real-time polymerase chain reaction (RT-PCR); Unexplained recurrent pregnancy loss (URPL).

\section{INTRODUCTION}

According to the American College of Obstetricians and Gynecologists [1], recurrent pregnancy loss is a disease, defined as two or more failed pregnancies. Recurrent pregnancy loss is one of the most frustrating and difficult areas of reproductive medicine because up to $50.0 \%$ of cases lack clearly defined etiology [2]. Common conditions that have been associated with recurrent pregnancy losses include uterine abnormalities, parental chromosomal aberrations, various endocrine disturbances and the presence of antiphospholipid antibodies [3,4]. Uncontrolled inflammatory response is thought an important mechanism underlying development of pregnancy complications such as unexplained pregnancy loss, preeclampsia, fetal growth restriction and death $[5,6]$. In the placenta, innate and adaptive immune responses influence the balance between anti- and pro-inflammatory decidual environments. These inflammatory changes are essential to various processes that are required for successful embryonic implantation, such as trophoblast invasion, angiogenesis and placental growth [7].

Pentraxins are a superfamily of evolutionarily conserved multifunctional pattern-recognition proteins that play roles in innate humoral immunity. Serum amyloid $\mathrm{P}$ component and $\mathrm{C}$ reactive protein constitute the short arm of the pentraxin superfamily. Pentraxin 3 (PTX3) is a prototype member of long arm that contains an additional 
$\mathrm{N}$-terminal domain. Unlike classic pentraxins made in the liver, PTX 3 is produced locally by a number of different cells such as mononuclear cells, dendritic cells and endothelial cells [8]. Pentraxin 3 is a multifunctional protein that interacts with various ligands, including elements of the complement system such as $\mathrm{Clq}$, factor $\mathrm{H}, \mathrm{C} 4$ binding protein, ficolins and mannose-binding lectin; growth factors such as fibroblast growth factor 2; adhesion molecules such as P-selectin; extracellular matrix components such as inter- $\alpha$-trypsin inhibitor; tumor necrosis factor-inducible gene- 6 and fibrin; apoptotic cells, and pathogens such as Klebsiella pneumoniae and Aspergillus fumigatus [9]. Hence, PTX3 plays important roles in innate immunity, complement activation, inflammation, matrix deposition, angiogenesis, vascular remodeling, platelet activation, antimicrobial resistance and tissue repair [8]. Pentraxin 3 also behaves as an acute-phase response protein that is rapidly induced from low blood levels in response to inflammation. Pentraxin 3 expression levels have also been correlated with disease severity under conditions of sepsis, acute myocardial infarction, atherosclerosis, autoimmune disorders and preeclampsia $[10,11]$.

During pregnancy, PTX3 is expressed in amniotic epithelial, chorionic mesoderm, terminal villous trophoblast and placental perivascular stromal tissues, and is increasingly expressed during pregnancy and peaks during labor $[12,13]$. However, no previous studies have shown PTX3 expression patterns in placental tissues of patients with unexplained recurrent pregnancy loss (URPL). Herein, we determined PTX3 expression levels in patients with URPL and compared these with those in a control group of subjects with a history of healthy live births.

\section{MATERIALS AND METHODS}

Study Subjects. In this retrospective case-control study, we evaluated placental tissues from 50 URPL patients and 50 healthy subjects who had full-term births between 2008 and 2014. Formalin-fixed/paraffin-embedded (FFPE) tissue samples that had been previously submitted for routine pathological examination were collected from the archives of the Department of Pathology, University of Pamukkale, Denizli, Turkey. The study protocol was approved by the Ethics Committee of Pamukkale University (No: 2014/1-1, Date: 01.07.2014), and all procedures were performed in conformance with the Declaration of Helsinki (2000).

The inclusion criteria for study subjects were the loss of more than two pregnancies in the presence of normal conceptus and parental karyotypes. The absence of anticardiolipin antibodies, lupus anticoagulating agents, uterine anomalies (determined via ultrasonography and hystero- salpingography), hormonal imbalances (due to polycystic ovary syndrome, diabetes and untreated thyroid disease), known autoimmune disease, such as lupus erythematosus or rheumatoid arthritis, thrombophilic abnormalities (indicated by Factor V Leiden thrombophilia and prothrombin $G 20210 A$ mutations), and histopathological placental anomalies, was confirmed in all included subjects. A total of 50 women met the inclusion criteria, and none had known diseases during sampling.

Placental tissues were collected from healthy women with single pregnancies; no history of pregnancy loss or pregnancy complications such as preeclampsia, eclampsia, preterm birth or intrauterine growth restriction, and no histopathological placental anomalies. No control subjects had undergone a cesarean section. Because placental PTX3 expression levels were reportedly highest at fullterm pregnancy, we made comparisons of PTX3 expression levels with those in full-term placentas from control subjects [12-14].

Sample Collection. All tissue sections were reevaluated, and optimal images of maternal placental areas, including decidual cells and trophoblasts, were collected. Cells were not analyzed separately because we do not have access to a laser microdissection instrument. Two $10 \mu \mathrm{m}-$ thick slices were cut from each FFPE block with disposable blades and placed in sterile $1.5 \mathrm{~mL}$ centrifuge tubes for total RNA extraction. Immunohistochemical (IHC) analyses of tissues from all study subjects were performed using 4 $\mu \mathrm{m}$-thick sections mounted on positively charged slides.

RNA Extraction and cDNA Synthesis. The tissue samples were deparaffinized by twice extracting with 1 $\mathrm{mL}$ of xylene for $10 \mathrm{~min}$., followed by rehydration through subsequent washes with $100.0,90.0$ and $70.0 \%$ ethanol diluted in RNase-free water. Total RNA was isolated from tissue samples using RNeasy® FFPE Kits (Qiagen $\mathrm{GmbH}$, Hilden, Germany) according to the manufacturer's instructions. The concentration and purity of the total RNA samples were determined using NanoDrop 2000c (Thermo Fisher Scientific, Wilmington, MA, USA). Total RNA samples of about $2 \mu \mathrm{g}$ were then incubated in a final reaction volume of $20 \mu \mathrm{L}$ containing the reagents for reverse transcription using a commercial kit (High Capacity cDNA Reverse Transcription Kit; Applied Biosystems, Foster City, CA, USA). cDNAs were stored at $-20^{\circ} \mathrm{C}$ until use as templates in quantitative real-time polymerase chain reactions (qRT-PCRs).

Quantitative Real-Time Polymerase Chain Reaction. Real-time PCR analyses were performed using the LightCycler 480 platform (Roche Diagnostics $\mathrm{GmbH}$, Penzberg, Germany) with the PCR primers and Universal ProbeLibrary (UPL) probes for PTX 3 and the internal reference gene ( $\beta$-actin) listed in Table 1. Primer sequences 
Table 1. Primer sequences and probes.

\begin{tabular}{|l|l|l|l|}
\hline Gene & Primer Sequences (5'>3') & UPL Probe & Amplicon size \\
\hline PTX3 & $\begin{array}{l}\text { F: GCTG GTG CTA GAG GAG CTG } \\
\text { R: GGA ATA AAA TAG CTG TTT CAC AAC CT }\end{array}$ & Probeno: \#23 (04686977001) & $106 \mathrm{bp}$ \\
\hline 3-actin & $\begin{array}{l}\text { F: CGA CAG GAT GCA GAA GGA G } \\
\text { R: AGG AGG AGC AAT GAT CTT GAT CT }\end{array}$ & Probeno: \#37 (04687957001) & $74 \mathrm{bp}$ \\
\hline
\end{tabular}

UPL: Universal ProbeLibrary; F: forward; $P T X 3$ : pentraxin 3; R: reverse; bp: base pair.

for PTX3 were 5'-CGG TGC TAG AGG AGC TG-3' and 5'-GGAATAAAA TAG CTG TTT CAC AAC CT-3', with UPL probe 23 ; and primer sequences for ACTB ( $\beta$-actin) were 5'-CGA CAG GAT GCA GAA GGA G-3' and 5'AGG AGG AGC AAT GAT CTT GAT CT-3', with an Universal ProbeLibrary (UPL) probe 37. Pentraxin 3 expression levels were determined in final reaction volumes of $20 \mu \mathrm{L}$ containing $0.5 \mu \mathrm{M}$ of each primer, $0.15 \mu \mathrm{M}$ of probe, $4 \mu \mathrm{L}$ of $\times 5$ LightCycler TaqMan Master Mix, $2 \mu \mathrm{L}$ of cDNA sample, and $13 \mu \mathrm{L}$ of PCR-grade water. The cycle conditions were 1 cycles at $95^{\circ} \mathrm{C}$ for $10 \mathrm{~min}$., followed by 45 cycles at $95{ }^{\circ} \mathrm{C}$ for 10 seconds, $60^{\circ} \mathrm{C}$ for 30 seconds and $72{ }^{\circ} \mathrm{C}$ for 1 second. Analyses were performed with negative control reaction mixtures containing PCR-grade water instead of DNA. Quantification of PTX3 mRNA expression levels was calculated based on the quantification cycle $(\mathrm{Cq})$ for each well, and normalized to $\beta$-actin as endogenous controls in both patient and control groups. Raw data were processed using LightCycler 480 software (Roche Diagnostics GmbH, Mannheim, Germany). The expression of PTX $3 \mathrm{mRNA}$ was calculated using the $\Delta \Delta \mathrm{Cq}$ method and compared with the expression in the control group. The difference was considered significant when the $p$ value was $<0.05$. The value was represented as the mean fold of RNA expression compared with the controls. $\beta$

Immunohistochemical Analyses. No monoclonal antibody against PTX3 was commercially available. Thus, IHC analyses were performed using polyclonal antibody against PTX3 (dilution, 1/250; Sigma-Aldrich, St. Louis, MO, USA). Tissue sections of $4 \mu \mathrm{m}$ in thickness were prepared from the URPL patients $(n=50)$ and controls ( $n$ $=50$ ). The sections were dehydrated at $60^{\circ} \mathrm{C}$ for 2 hours and were then stained using an automated staining system (Ventana Medical Systems Inc., Tuczon, AZ, USA) according to the manufacturer's instructions. Briefly, antigen retrieval were performed by an acidic citrate buffer-based solution (CC2; Ventana Medical Systems Inc.) for 1 hour. The tissue sections were incubated with anti-PTX 3 antibody for 1 hour at $37^{\circ} \mathrm{C}$. The slides were then counterstained with hematoxylin II and bluing reagent (Ventana Medical Systems Inc.) for $4 \mathrm{~min}$., then dehydrated in a graded series of ethyl alcohol and xylene baths. Negative controls were achieved by substituting the primary antibody with phosphate-buffered saline, and PTX3-immuno- reactive cells were counted under a light microscope with a $\times 40$ objective lens.

Cytoplasmic PTX3 staining intensities and percentages of positive cells were evaluated and recorded as 0 (no staining), 1 (1.0-10.0\% staining of the cells), 2 (11.0$25.0 \%$ staining of the cells), 3 (26.0-50.0\% staining of the cells) or 4 ( $>50.0 \%$ staining of the cells) as described previously [15].

Statistical Analyses. Statistical analyses were performed using the Statistical Package for the Social Sciences (SPSS $\otimes$ ), version 23.0 (IBM SPSS Inc., Armonk, NY, USA). Data were expressed as mean values \pm standard deviations (SDs). Differences between patient and control groups were identified using Mann-Whitney $U$ test and were considered significant when the $p$ value was $<0.05$. Pearson correlation was used to determine the correlation between the PTX3 expression levels obtained by IHC and qRT-PCR.

\section{RESULTS}

The characteristics of the URPL patients are shown in Table 2. Patient and control groups did not differ significantly in terms of age ( $p=0.819)$, and $30(60.0 \%), 17$ $(34.0 \%)$, and three (6.0\%) URPL patients had zero, one, and two live births, respectively. Numbers of previous miscarriages and gestational weeks of current miscarriages (mean $\pm \mathrm{SD}$ ) were $2.34 \pm 0.77$ and $10.16 \pm 3.58$, respectively.

The present qRT-PCR analyses were successful for all samples, and PTX3 mRNA expression in the patient group was 116-fold higher than that in the control group ( $p=0.0001$; Figure 1). In the patient group, no significant correlations were found between PTX3 mRNA expression levels and maternal ages, numbers of live births, previous numbers of miscarriages, and gestational weeks of miscarriage. The URPL patients were divided into the patients with no previous live births and the patients with previous live births, and PTX3 mRNA expression levels did not differ significantly between the two groups. However, PTX3 mRNA expression levels of the patients with no live births were significantly elevated in comparison with PTX3 mRNA expression levels of URPL patients and control subjects with previous live births ( $p=0.0001$; Figure 2). 
Table 2. Unexplained recurrent pregnancy loss and control group characteristics.

\begin{tabular}{|l|l|l|l|l|l|}
\hline Parameters & \multicolumn{1}{|c|}{ Mean \pm SD } & \multicolumn{1}{|c|}{$\begin{array}{c}\text { Mean Values } \\
(\mathrm{min}-\mathrm{max})\end{array}$} & \multicolumn{1}{|c|}{ Mean \pm SD } & \multicolumn{1}{c|}{$\begin{array}{c}\text { Mean Values } \\
(\mathrm{min}-\mathrm{max})\end{array}$} & $p$ Value \\
\hline Age (years) & $29.76 \pm 5.04$ & $29(22-40)$ & $29.34 \pm 4.51$ & $29(19-41)$ & 0.819 \\
\hline $\begin{array}{l}\text { Number of previous } \\
\text { miscarriages }\end{array}$ & $2.34 \pm 0.77$ & $2(2-5)$ & - & - & - \\
\hline $\begin{array}{l}\text { Gestational week of } \\
\text { current miscarriage }\end{array}$ & $10.16 \pm 3.58$ & $9(5-20)$ & - & - & - \\
\hline $\begin{array}{l}\text { Number of previous } \\
\text { live births }\end{array}$ & $0.46 \pm 0.61$ & $0(0-2)$ & $1.68 \pm 0.74$ & $2(1-4)$ & 0.0001 \\
\hline $\begin{array}{l}\text { PTX3 mRNA } \\
\text { expression levels }\end{array}$ & $0.0014 \pm 0.0059$ & $0.0001(0.000-0.418)$ & $0.000012 \pm 0.000038$ & $0.000002(0.000-0.000264)$ & 0.0001 \\
\hline
\end{tabular}

UPRL: Unexplained recurrent pregnancy loss; SD: standard deviation; min-max, minimum-maximum.

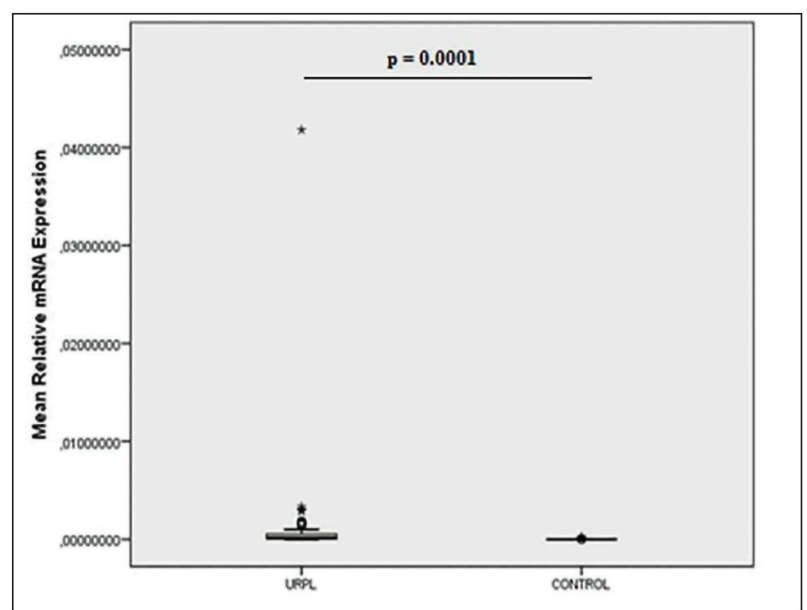

Figure 1. Mean $P T X 3 \mathrm{mRNA}$ expression normalized for $\beta$-actin mRNA expression in the placental tissues from URPL patients and control subjects. The small circles mean the outlier values and the asterisks $\left({ }^{*}\right)$ indicate extreme values.

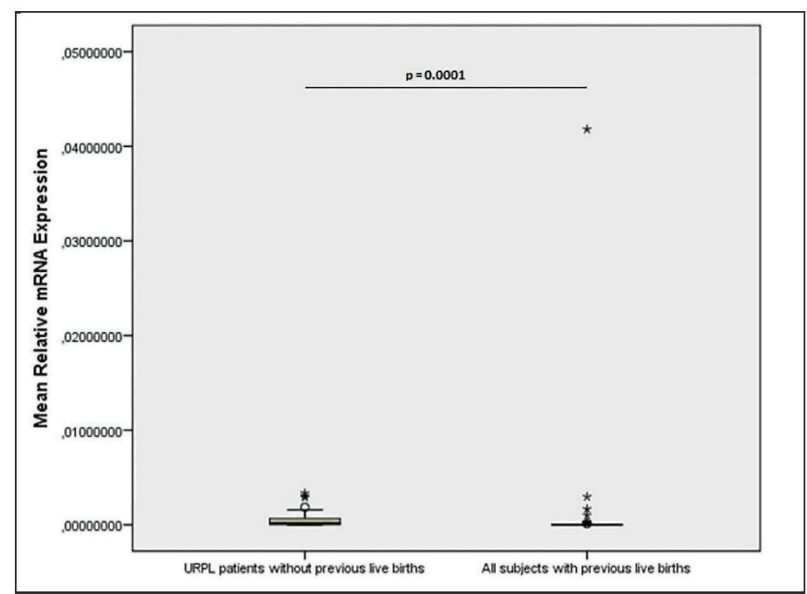

Figure 2. Mean $P T X 3 \mathrm{mRNA}$ expression normalized for $\beta$-actin mRNA expression in the placental tissues from URPL patients without live birth, all women (URPL patients and controls) with previous live births. The small circles mean the outlier values and the asterisks $\left({ }^{*}\right)$ indicate extreme values.
Immunohistochemistry analysis showed that the PTX3 protein expression was widely distributed in the villous and extravillous cytotrophoblast, and also in the

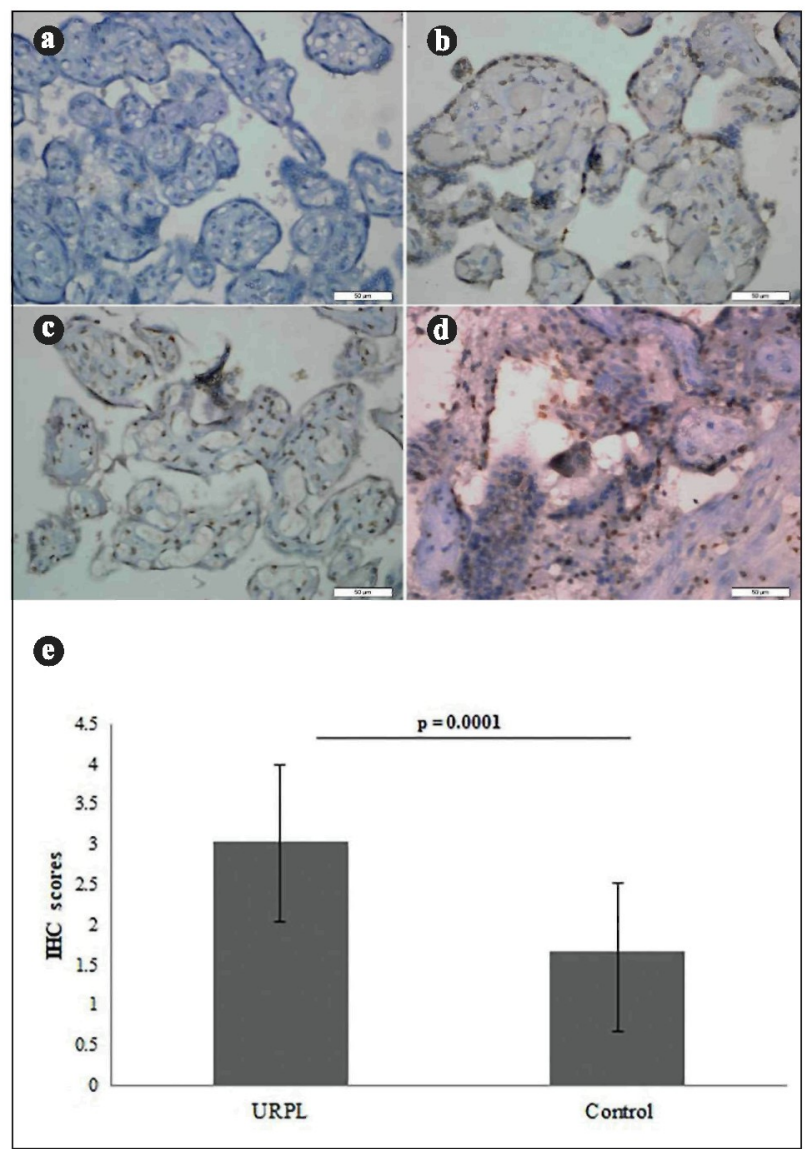

Figure 3. Representative images of PTX3 immunhistochemistry staining in the placental tissues derived from control subjects ( $a$ and $b$ ) and URPL patients ( $c$ and d). Pentraxin 3 is exclusively expressed in the cytoplasm. Positive staining is shown in brown. Subjects were semi-quantified as score 1 (a), score 2 (b), score 3 (c), score 4 (d) (original magnification, X400). (e) Immunhistochemistry scores for PTX 3 expression was significantly higher in URPL patients than the control subjects. Values are expressed as means \pm SDs. 
stromal and endothelial cells. Similar tissue distribution was observed in both URPL patients and control subjects. The PTX 3 protein expression was scored as 1 in four, 2 in 10,3 in 16 and 4 in 20 URPL patients, whereas the same scores of $1,2,3$ and 4 were assigned to 28,13 , seven and two control subjects, respectively. Figure 3(a) to 3(d) shows representative images of the IHC staining scores. As in PTX3 mRNA expression analyses, protein expression levels differed significantly between URPL patients and controls ( $p=0.0001)$ [Figure 3(e)]. We also compared the results of both methodologies, and there was a weak positive correlation between PTX3 mRNA and protein expression levels $(p=0.023 ; r=0.227)$.

\section{DISCUSSION}

The multifunctional protein PTX3 is a well-known pattern recognition receptor that interacts with multiple ligands, including $\mathrm{Clq}$, fibroblast growth factor 2 , and P-selectin. The PTX3 is also reportedly involved in the pathogenesis of disorders such as atherosclerosis and inflammatory rheumatic disease and in pregnancy-related problems such as preeclampsia and preterm births $[16,17]$. Several previous studies showed a significant overexpression of PTX 3 depending on disease severity in women with preeclampsia $[18,19]$. Furthermore, when maternal plasma PTX3 levels were measured during the early weeks of pregnancy, subjects who later developed preeclampsia were found to have significantly higher levels than those who did not develop preeclampsia [20-22]. Therefore, PTX3 may be considered an early marker of placental dysfunction.

Incorrect decidualization and implantation was previously observed in $\mathrm{Ptx}^{3^{--}}$mice, suggesting that PTX3 plays an important role in blastocyst invasion during endometrial differentiation and implantation stages [23]. The $P T X 3$ is one of the few genes that are induced in fetal implantation areas and is expressed most strongly from decidualized stromal cells following inflammatory events that are mediated by trophoblasts, natural killer cells and antigenpresenting cells $[24,25]$. To the best of our knowledge, the present study is the first to investigate PTX 3 mRNA and protein levels in placental tissues from women with URPL using qRT-PCR and IHC.

Recent studies indicate that the highest PTX3 levels in plasma occur during labor $[13,14,26]$. Moreover, significant differences in plasma PTX3 levels were observed between women with differing types of delivery. Specifically, significantly higher PTX3 levels were observed after vaginal deliveries than after cesarean deliveries [27,28]. Therefore, we excised tissue sections containing trophoblast and decidual cells from the maternal side of placenta after full-term healthy pregnancies and excluded women with cesarean deliveries from the control group.

In a 2012 publication, Ibrahim et al. [29] demonstrated the relationship between PTX3 levels and recurrent miscarriage. They compared plasma PTX 3 concentrations between patients with primary recurrent miscarriages and pregnant women at the same gestational week and found significantly higher plasma PTX3 levels in the miscarriage group and a positive correlation with numbers of previous miscarriages. Herein, we determined PTX3 expression in placental tissues, and similar to previous studies, we observed 116-fold higher PTX3 expression in the patient group than in the control group. We did not, however, observe significant correlations with age, numbers of previous miscarriages, gestational week of the current miscarriage, or the number of previous live births. Nonetheless, PTX 3 expression levels differed significantly between patients who had no live births and women with previous live births. We suggest that aberrant inflammatory signaling contributes to URPL and involves PTX3. In agreement with this hypothesis, the PTX3 ligand Clq reportedly plays key roles in trophoblast invasion, spiral artery remodeling, and normal placentation [30,31], and abnormal distributions of $\mathrm{Clq}$ have been associated with adverse pregnancy outcomes such as miscarriage, preterm delivery and pre-eclampsia [32].

In the present study, we found that PTX3 expression was dramatically increased in placental tissues from patients with URPL. Hence, immune dysregulation may be among the etiological factors for URPL, but further functional studies are needed to examine the interactions between PTX3 and other immunological signaling molecules that are stimulated under the conditions of URPL.

\section{ACKNOWLEDGMENTS}

Selcan Zeybek and Emre Tepeli, Department of Medical Genetics, Denizli, Turkey, Basak Yildirim, Department of Gynaecology and Obstetrics, Denizli, Turkey, were the faculty staff at the Faculty of Medicine, Pamukkale University, Denizli, Turkey, when the present study was carried out. The authors would like to thank Dr. Metin Akbulut (faculty staff at the Department of Pathology, Faculty of Medicine, Pamukkale Unitersity, Denizli, Turkey, when the present study was carried out), for his technical contribution to the immunohistochemistry staining.

Declaration of Interest. The authors report no conflicts of interest. The authors alone are responsible for the content and writing of this article.

Funding. This research was supported by Pamukkale University Scientific Research Unit(Grant \#2014TPF014). 


\section{REFERENCES}

1. Practice Committee of the American Society for Reproductive Medicine. Definitions of infertility and recurrent pregnancy loss. Fertil Steril. 2013; 99(1): 63.

2. Jaslow CR, Carney JL, Kutteh WH. Diagnostic factors identified in 1020 women with two versus three or more recurrent pregnancy losses. Fertil Steril. 2010; 93(4): 1234-1243.

3. Ford HB, Schust DJ. Recurrent pregnancy loss: Etiology, diagnosis, and therapy. Rev Obstet Gynecol. 2009; 2(2): 76-83.

4. Jeve YB, Davies W. Evidence-based management of recurrent miscarriages. J Hum Reprod Sci. 2014; 7(3): 159-169.

5. Christiansen OB. Reproductive immunology. Mol Immunol. 2013; 55(1): 8-15.

6. Kalagiri RR, Carder T, Choudhury S, Vora N, Ballard $\mathrm{AR}$, Govande $\mathrm{V}$, et al. Inflammation in complicated pregnancy and its outcome. Am J Perinatol. 2016; 33(14): 1337-1356.

7. Mor G, Cardenas I, Abrahams V, Guller S. Inflammation and pregnancy: The role of the immune system at the implantation site. Ann NY Acad Sci. 2011; 1221(1): 80-87.

8. Doni A, Garlanda C, Mantovani A. Innate immunity, hemostasis and matrix remodeling: PTX3 as a link. Semin Immunol. 2016; 28(6): 570-577.

9. Bottazzi B, Doni A, Garlanda C, Mantovani A. An integrated view of humoral innate immunity: pentraxins as a paradigm. Annu Rev Immunol. 2010; 28: 157-183.

10. Garlanda C, Maina V, Cotena A, Moalli F. The soluble pattern recognition receptor pentraxin-3 in innate immunity, inflammation and fertility. J Reprod Immunol. 2009; 83(1-2): 128-133.

11. Casula M, Montecucco F, Bonaventura A, Liberale $\mathrm{L}$, Vecchié A, Dallegri F, et al. Update on the role of Pentraxin 3 in atherosclerosis and cardiovascular diseases. Vascul Pharmacol. 2017; 99: 1-12.

12. Larsson A, Palm M, Helmersson J, Axelsson O. Pentraxin 3 values during normal pregnancy. Inflammation. 2011; 34(5): 448-451.

13. Lekva T, Michelsen AE, Bollerslev J, Norwitz ER, Aukrust $\mathrm{P}$, Henriksen $\mathrm{T}$, et al. Low circulating pentraxin 3 levels in pregnancy is associated with gestational diabetes and increased apoB/apoA ratio: $\mathrm{A}$ 5-year follow-up study. Cardiovasc Diabetol. 2016; 15: 23.
14. Rovere-Querini P, Antonacci S, Dell'Antonio G, Angeli A, Almirante G, Cin ED. Plasma and tissue expression of the long pentraxin 3 during normal pregnancy and preeclampsia. Obstet Gynecol. 2006; 108(1): 148-155.

15. Willeke F, Assad A, Findeisen P, Schromm E, Grobholz R, von Gerstenbergk B, et al. Overexpression of a member of the pentraxin family (PTX3) in human soft tissue liposarcoma. Eur J Cancer. 2006; 42(15): 2639-2646.

16. Jaillon $S$, Bonavita $E$, Gentile $S$, Rubino $M$, Laface I, Garlanda C, et al. The long pentraxin PTX3 as a key component of humoral innate immunity and a candidate diagnostic for inflammatory diseases. Int Arch Allergy Immunol. 2014; 165(3): 165-178.

17. Giacomini A, Ghedini GC, Presta M, Ronca, R. Long pentraxin 3: A novel multifaceted player in cancer. Biochim Biophys Acta. 2018; 1869(1): 53-63.

18. Cozzi V, Garlanda C, Nebuloni M, Maina V, Martinelli A, Calabrese S, et al. PTX3 as a potential endothelial dysfunction biomarker for severity of preeclampsia and IUGR. Placenta. 2012; 33(12): 1039-1044.

19. Zhou P, Luo X, Qi HB, Zong WJ, Zhang H, Liu DD, et $a l$. The expression of pentraxin 3 and tumor necrosis factor- $\alpha$ is increased in preeclamptic placental tissue and maternal serum. Inflamm Res. 2012; 61(9): 1005 1012.

20. Akolekar R, Casagrandi D, Livanos P, Tetteh A, Nicolaides KH. Maternal plasma pentraxin 3 at 11 to 13 weeks of gestation in hypertensive disorders of pregnancy. Prenat Diagn. 2009; 29(10): 934-938.

21. Baschat AA, Kasdaglis TL, Aberdeen GW, Turan OM, Kopelman JL, Atlas R, et al. Serum pentraxin-3 levels at 11 to 14 weeks' gestation: Association with maternal and placental characteristics. Am J Obstet Gynecol. 2009; 201(3): 298.e1-298.e6.

22. Cetin I, Cozzi V, Papageorghiou AT, Maina V, Montanelli A, Garlanda C, et al. First trimester PTX3 levels in women who subsequently develop preeclampsia and fetal growth restriction. Acta Obstet Gynecol Scand. 2009; 88(7): 846-849.

23. Tranguch S, Chakrabarty A, Guo Y, Wang H, Dey SK. Maternal pentraxin 3 deficiency compromises implantation in mice. Biol Reprod. 2007; 77(3): 425432.

24. Popovici RM, Betzler NK, Krause MS, Luo M, Jauckus J, Germeyer A, et al. Gene expression profiling of human endometrial-trophoblast interaction in a coculture model. Endocrinology. 2006; 147(12): $5662-5675$ 
25. Hess AP, Hamilton AE, Talbi S, Dosiou C, Nyegaard $M$, Nayak $N$, et al. Decidual stromal cell response to paracrine signals from the trophoblast: Amplification of immune and angiogenic modulators. Biol Reprod. 2007; 76(1): 102-117.

26. Cruciani L, Romero R, Vaisbuch E, Kusanovic JP, Chaiworapongsa T, Mazaki-Tovi S, et al. Pentraxin 3 in maternal circulation: An association with preterm labor and preterm PROM, but not with intra-amniotic infection/inflammation. J Matern Fetal Neonatal Med. 2010; 23(10): 1097-1105.

27. Assi F, Fruscio R, Bonardi C, Ghidini A, Allavena $P$, Mantovani A, et al. Pentraxin 3 in plasma and vaginal fluid in women with preterm delivery. Br J Obstet Gynaecol. 2007; 114(2): 143-147.

28. Jo YS, Cheon JY, Ahn JW, Kim Y, Lee GSR. Pentraxin 3 and lipid profile status in pregnancy. J Obstet Gynaecol. 2017; 37(3): 727-730.
29. Ibrahim MI, Harb HM, Ellaithy MI, Elkabarity RH, Abdelgwad MH. First trimester assessment of pentraxin-3 levels in women with primary unexplained recurrent pregnancy loss. Eur J Obstet Gynecol Reprod Biol. 2012; 165(1): 37-41.

30. Agostinis C, Bulla R, Tripodo C, Gismondi A, Stabile $\mathrm{H}$, Bossi $\mathrm{F}$, et al. An alternative role of $\mathrm{Clq}$ in cell migration and tissue remodeling: Contribution to trophoblast invasion and placental development. J Immunol. 2010; 185(7): 4420-4429.

31. Kouser L, Madhukaran SP, Shastri A, Saraon A, Ferluga J, Al-Mozaini M, et al. Emerging and novel functions of complement protein C1q. Front Immunol. 2015; 6: 317.

32. Singh J, Ahmed A, Girardi G. Role of complement component $\mathrm{Clq}$ in the onset of preeclampsia in mice. Hypertension. 2011; 58(4): 716-724. 